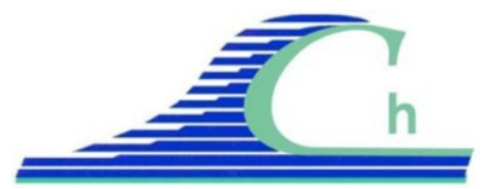

XII ${ }^{\text {ìmes }}$ Journées Nationales Génie Côtier - Génie Civil

Cherbourg, 12-14 juin 2012

DOI:10.5150/jngcgc.2012.093-C @ Editions Paralia CFL

disponible en ligne - http://www.paralia.fr - available online

\title{
Cartographie des habitats intertidaux des sites Natura 2000 des Pertuis charentais : complémentarité des approches inventaire terrain, télédétection et SIG
}

\author{
Pauline CAJERI ${ }^{1}$, Cécile CURTI ${ }^{1}$, Virginie LAFON ${ }^{2}$, Ronan LAUNAY $^{3}$, \\ Pierre-Guy SAURIAU ${ }^{1}$
}

\section{LIENSs, CNRS, Université de la Rochelle, UMR 7266 CNRS - ULR,} 2 rue Olympe de Gouges, 17000 La Rochelle, France. pauline.cajeri@univ-Ir.fr ; cecile.curti@univ-Ir.fr ; pierre-guy.sauriau@univ-lr.fr

2. GEO-Transfert, UMR 5805 EPOC - Université Bordeaux 1, Avenue des facultés, 33405 Talence cedex, France.

v.lafon@epoc.u-bordeaux1.fr

3. CREOCEAN, rue Charles Tellier, Zone Technocéan, 17000 La Rochelle, France. launay@creocean.fr

\section{Résumé :}

A la demande de l'Agence des Aires Marines Protégées, un inventaire cartographique des habitats des sites Natura 2000 en mer de l'estuaire de la Gironde et des Pertuis charentais a été réalisé. L’objectif est d'établir un état initial de référence des milieux naturels afin de contribuer aux connaissances nécessaires à leur gestion durable. S'inspirant des protocoles REBENT et MESH, trois méthodes complémentaires ont été combinées pour cartographier les habitats intertidaux : inventaires terrain avec relevés GPS différentiel, télédétection couplant analyse d'ortho littorales et de scènes du satellite KOMPSAT-2 puis intégration sous Système d’Information Géographique. Cette combinaison exploite les avantages de chaque méthode et optimise le rendu cartographique basé sur la typologie EUNIS. Les inventaires terrain donnent une description précise de la succession des habitats et sont indispensables pour géolocaliser les habitats d'espèces (hermelles, huîtres, zostères). Les structures délimitées sur photographies aériennes et validées par les inventaires terrain sont interprétées à partir de scènes satellite soumises à classification hiérarchique, permettant une discrimination en termes de nature de sol, type de végétation et présence de mares. La compilation cohérente de ces sources d'information est réalisée sous SIG en respectant les contraintes d'échelle et de topologie. A titre d'exemple, la cartographie des habitats intertidaux de l'île d'Aix est illustrée. Sont présents, à minima, 15 habitats élémentaires y compris ceux d'espèces patrimoniales (hermelles, zostères). Le flanc nord-ouest de l'île se singularise par de remarquables mosaïques d'habitats rocheux.

\section{Mots-clés :}

Natura 2000 - Estran -Vérité terrain - Télédétection - SIG - Cartographie - EUNIS

\section{Introduction}


Protection et conservation du patrimoine naturel sont aujourd'hui des enjeux majeurs du développement durable à l'échelle planétaire. La Convention sur la diversité biologique consacrée par le troisième Sommet de la Terre (Rio de Janeiro 1992) est à l'origine de stratégies concertées de conservation de la biodiversité ainsi que d'objectifs hiérarchisés de gestion (MARTY et al., 2005). Les programmes de l’Union Européenne sont ainsi déclinés aux échelles nationales et régionales au titre des directives Natura 2000, Directive Oiseaux de 1979 puis Directive Habitats Faune Flore de 1992. Un réseau de sites représentatifs et identifiés pour la rareté de leurs espèces faune-flore et les fonctionnalités de leurs habitats a ainsi été crée. Dans le contexte français, le réseau Natura 2000 initialement terrestre (PINTON et al., 2007) a été complété depuis 2008 par la désignation de sites Natura 2000 en mer. L’Agence des Aires Marines Protégées coordonne depuis 2009 à l'échelle nationale des inventaires biologiques et des analyses écologiques de ces sites avec pour objectif de rassembler les connaissances nécessaires à leur gestion. L'analyse de ces milieux naturels se base sur des outils comme les inventaires d'espèces et les cartographies d'habitats. Ces méthodologies adaptées au paradigme Natura 2000 ont été éprouvées en milieu terrestre (HARDEGEN et al., 2001) mais leur application au domaine marin nécessite des adaptations pour tenir compte de spécificités structurelles et fonctionnelles intrinsèques (DAUVIN, 1997 ; BENSETTITI et al., 2004). Ont ainsi été dressées des cartographies sectorielles lors des programmes REBENT (ROLLET et al., 2005 ; GUILLAUMONT et al., 2009) et MESH (PROJET MESH, 2008) porteurs de mises au point méthodologiques. Nous développons la stratégie mise en œuvre, les méthodes et outils utilisés pour dresser une cartographie des habitats marins intertidaux des Pertuis charentais selon la typologie EUNIS (DAVIES et al., 2004). Est présentée en guise d’illustration le cas de l'île d’Aix (Charente-Maritime) connue pour la richesse et l'originalité de sa faune et de sa flore marine (TARDY \& TARDY, 1977).

\section{Méthodologie}

\subsection{Zone d'étude et stratégie d'analyse}

La zone d'étude de l'estuaire de la Gironde aux Pertuis charentais s'étend sur plus de $6440 \mathrm{~km}^{2}$ (figure 1A). Elle correspond au périmètre du futur parc naturel marin (http://www.aires-marines.fr/gironde-pertuis.html) qui inclut neuf zones de protection spéciale (ZPS) et huit sites d'intérêt communautaire (SIC). Le SIC FR5400430 Vallée de la Charente (basse vallée) englobe l'île d'Aix (figure 1B). Notre stratégie d'analyse pour aboutir à la cartographie des habitats marins intertidaux a combiné : des inventaires terrain positionnés après une première analyse des ortho littorales, la télédétection couplant des scènes du satellite KOMPSAT-2 analysées par arbres hiérarchiques et des ortho littorales 2000 (C IGN) interprétées grâce aux vérités terrain. 


\section{XII ${ }^{\text {èmes }}$ Journées Nationales Génie Côtier - Génie Civil \\ Cherbourg, 12-14 juin 2012}

$\mathrm{Au}$ final l'intégration de ces informations spatialisées se fait sous Système d’Information Géographique (ArcGIS 10, (C) ESRI).

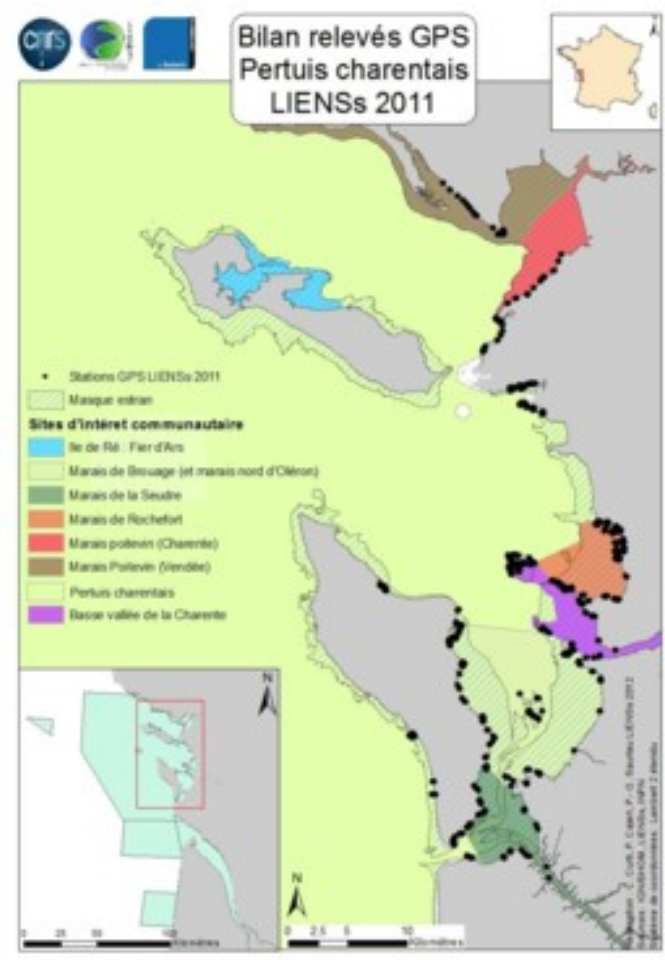

A

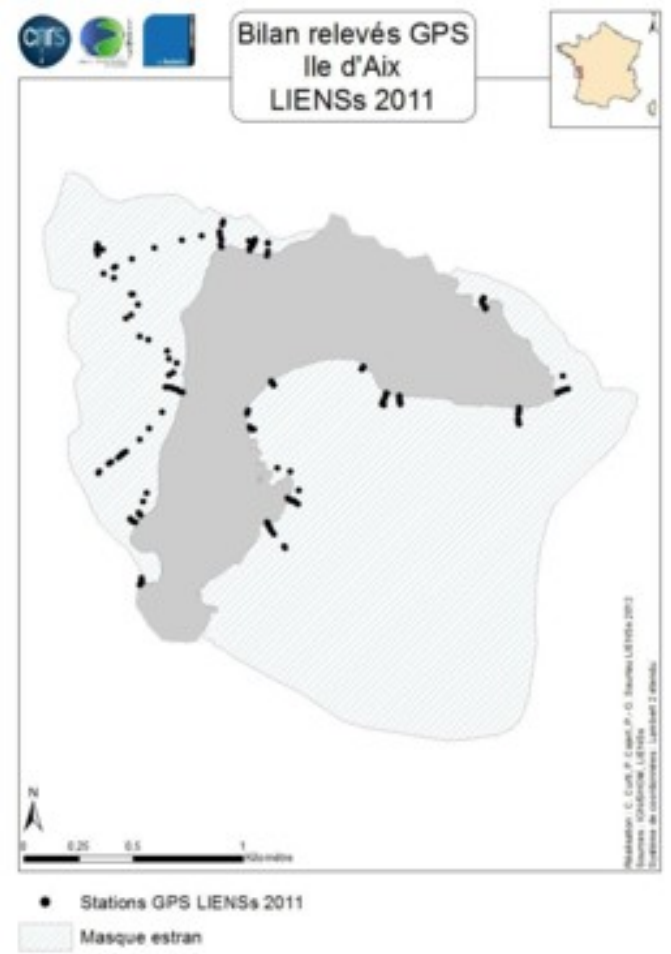

B

Figure 1. Zone d'étude avec emprise générale, contours des huit sites d'intérêt communautaire (SIC), limite haute et limite basse de l'estran et transects des vérités terrain (A) ; détails sur l'île d'Aix de la position des transects et stations (B)

\subsection{Inventaire terrain}

Une analyse préalable des ortho littorales réalisées à basse mer a été systématiquement menée de façon à positionner les inventaires terrain (transects de stations théoriques) selon la nature de sol (roche, sédiment, flaque), la topographie des estrans et la présence supposée de végétation (macroalgues et phanérogames marines). Les parcours in situ sur ces transects théoriques ont été effectués avec un GPS différentiel Trimble GeoXT 2005 équipé du logiciel TerraSync 3.21. Le post traitement a été réalisé sous Pathfinder office 4.10 afin d'obtenir une précision submétrique.

Le long des transects, les changements d'habitat sont repérés par un "point limite" dont le positionnement GPS est enregistré simultanément à une photographie d’ensemble. A l'intérieur de chaque habitat un "point station" est acquis accompagné de notes sur la table attributaire du GPS et de photographies d'ensemble et de détail de la station (espèces, substrat) sur un quadrat de $0,1 \mathrm{~m}^{2}$. Au total 1212 relevés (point limite et point station) ont été réalisés en 2011 par l'équipe LIENSs (figure 1A) dont 179 pour l'île d'Aix réalisés les 22 et 23 mars 2011 (figure 1B). Ces observations sont complétées si 
nécessaire par des prélèvements faunistiques et sédimentaires, ces derniers étant interprétés en cohérence avec les cartes biosédimentaires disponibles (HILY, 1976).

\subsection{Télédétection et analyse des scènes KOMPSAT}

Treize scènes KOMPSAT-2 ont été acquises pour couvrir la zone d'étude. D'une résolution au sol de $4 \mathrm{~m}$, elles incorporent 4 bandes spectrales dans le bleu $(0,45-$ $0,52 \mu \mathrm{m})$, le vert $(0,52-0,60 \mu \mathrm{m})$, le rouge $(0,63-0,69 \mu \mathrm{m})$ et le proche infrarouge $(0,76-0,90 \mu \mathrm{m})$. La dalle Marsilly-Fouras datée du 3 janvier 2011 inclue l'île d'Aix. Chaque scène est géo-référencée en Lambert 93 à l'aide des Scan 25 IGN (GEOTransfert) puis classée sous ENVI (@ ITT VIS) à l'aide d'un arbre hiérarchique adapté à la date d'acquisition (figure 2). Dans ce but, les levés DGPS des inventaires terrains ont été superposés aux images et les tables attributaires associées ont été analysées afin de déduire les signatures spectrales typiques de milieux distincts. Les arbres hiérarchiques sont ainsi constitués chacun d'une vingtaine de nœuds permettant de discriminer les différents types de milieux. La discrimination est basée sur des combinaisons de rapports de canaux. L'arbre de décision pour l'île d'Aix écarte à un premier niveau, les zones d'ombre (Ombre) et d'eaux permanentes (Eau) du bas de l'estran des zones d'intérêt (figure 2). Celles-ci identifient les mares permanentes (MP), les ceintures d'algues (Alg ou Algues), les substrats rocheux (RC), les blocs (BL), les galets (GT), les milieux sableux secs (SS), humides (SH) et de rétention (Rétention), les sables vaseux (SV), vases sableuses (VS) et vases pures (VV).

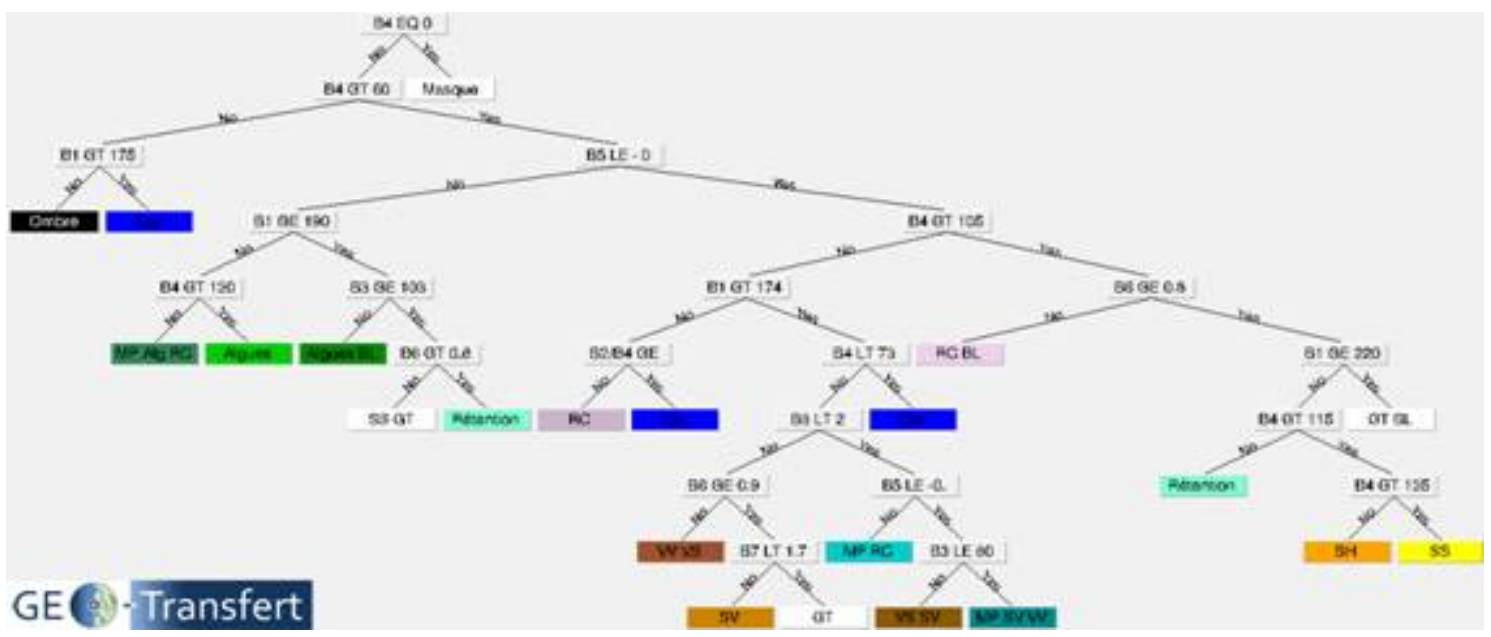

Figure 2. Arbre hiérarchique utilisé pour la scène KOMPSAT incluant l'’̂le d'Aix. Les sigles encadrés en couleur sont explicités dans le texte.

\subsection{Typologie EUNIS}

La typologie retenue pour la cartographie des habitats est la classification EUNIS (DAVIS et al., 2004) référence européenne de classification des habitats pour les domaines terrestres, dulçaquicoles et marins (http://eunis.eea.europa.eu/habitats.jsp) 


\section{XII ${ }^{\text {èmes }}$ Journées Nationales Génie Côtier - Génie Civil \\ Cherbourg, 12-14 juin 2012}

dans sa version 2008 (http://inpn.mnhn.fr/telechargement/referentiels/habitats\#eunis). Cette typologie repose sur un classement hiérarchique des habitats permettant, pour un domaine (niveau 1), d'accéder à des niveaux de précision allant de la simple distinction entre les types rocheux ou meuble (niveau 2), intégrant d'une part, le mode d'exposition et le type de substrat (niveau 3), puis, d'autre part, la notion de groupement fonctionnel d'habitats (niveau 4) jusqu'à l'identification précise des peuplements benthiques définis par la présence d'espèces dominantes ou de groupes d'espèces caractéristiques (niveaux 5 et 6). Le niveau 4 de la typologie EUNIS sera utilisé. En cas d'imbrication d'habitats élémentaires à des échelles non compatibles avec le rendu cartographique au $1 / 10000^{\text {ème }}$, des mosaïques d'habitats sont utilisées.

\subsection{Intégration des données sous SIG}

La zone intertidale à cartographier est délimitée en amont par le trait de côte HistoLitt® V1 (laisse des plus hautes mers) et en aval par la laisse des plus basses mers (৫) SHOM). L'intégration sous SIG a pour but de superposer les vérités terrain interprétées en habitats EUNIS et organisées ligne par ligne en fichiers Excel (@ Microsoft) aux résultats d'analyse des scènes KOMPSAT, tout en gardant la cohérence géographique des structures visibles sur les orthophotographies. Les données spatiales et aériennes sont complémentaires pour interpoler la continuité des habitats entre les transects des inventaires terrain. Les analyses des scènes KOMPSAT délivrent des fichiers de formes validés par les vérités terrain. Leur aspect "en marches d'escalier" basé sur l'agglomération de pixels ( 4 x $4 \mathrm{~m}$ ) est cependant peu réaliste. Sous SIG, les contours des polygones d'habitats sont créés manuellement à partir des ortho littorales 2000 afin d'obtenir une cartographie respectant règles de topologie et cohérence d'échelle (figure $3 A)$. L'échelle de numérisation est au $1 / 1000^{\text {ème }}$. La cartographie définitive est associée à une table attributaire renseignée en habitats EUNIS de niveau 4. L'habitat d'espèces "hermelles" est traité en partie en couche de points. L'habitat herbier à Zostera noltii, dont les emprises surfaciques ont été pour la plupart déterminées lors de contrôles de surveillance surfacique DCE (AUBY et al., 2010), est intégré à la couche de polygones par remplacement. Le cadastre conchylicole (zone de culture d'huîtres et de moules) est considéré comme une pression anthropique.

\section{Résultats et discussion}

L'île d'Aix (figure 3B) sert d'illustration à la démarche utilisée sur l'ensemble des estrans des Pertuis charentais (figure 1A)

\subsection{Carte des habitats intertidaux de l'île d'Aix en typologie EUNIS niveau 4}

La cartographie (figure 3B; tableau 1) intègre des habitats élémentaires, par exemple les bancs de moules et de cirripèdes (A1.11), des mosaïques d'habitats littoraux ainsi que des habitats comme "huîtres sur roches" qui sont en demande de création de code car 
absents de la typologie EUNIS (GUILLAUMONT et al., 2009 ; BAJJOUK et al., 2010).

Les habitats les plus représentés sont les vases, vase marine intertidale (A2.33) et vase sableuse infralittorale (A5.33) avec une couverture totale de 2,455 $\mathrm{km}^{2}$ (figure 3B, tableau 1). Bien que situés à proximité de l'estuaire de la Charente, ces habitats sont marins avec des normales décennales de salinité supérieures à 30 (LE MOINE, comm. pers., 2011). Ils se situent en continuité des vasières intertidales de la baie d'Yves et des vases sableuses infralittorales à Nucula nitidosa (mollusque bivalve) et Sternaspis scutata (annélide, polychètes) dominantes depuis l'Anse de l'Aiguillon au Nord jusqu'aux roches des Palles en baie de Marennes-Oléron (HILY, 1976).

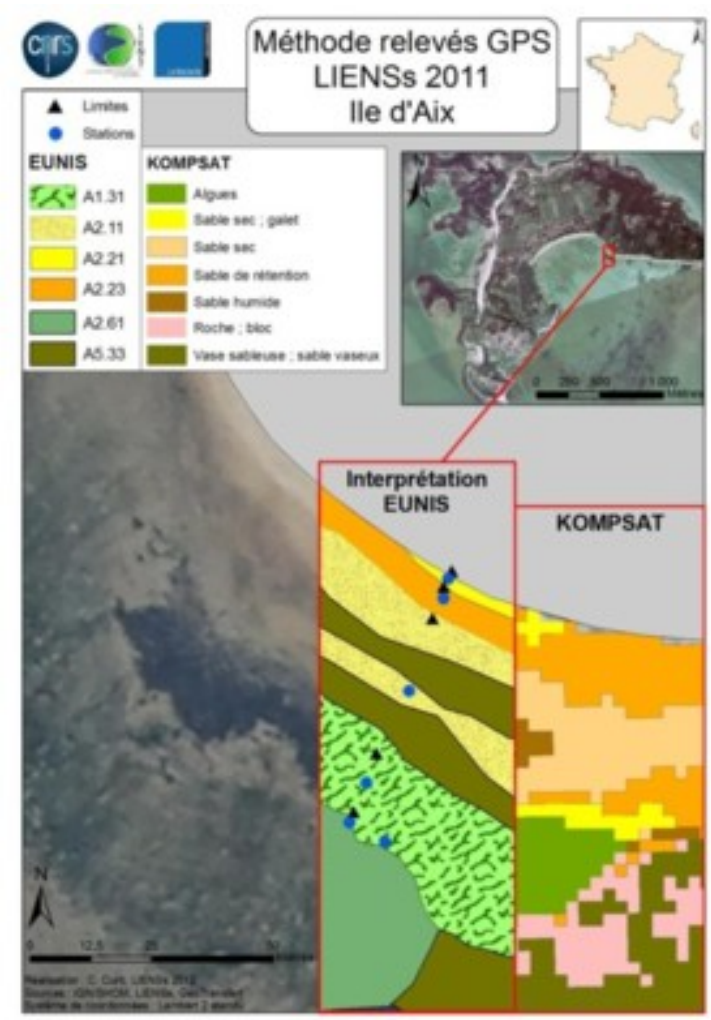

A

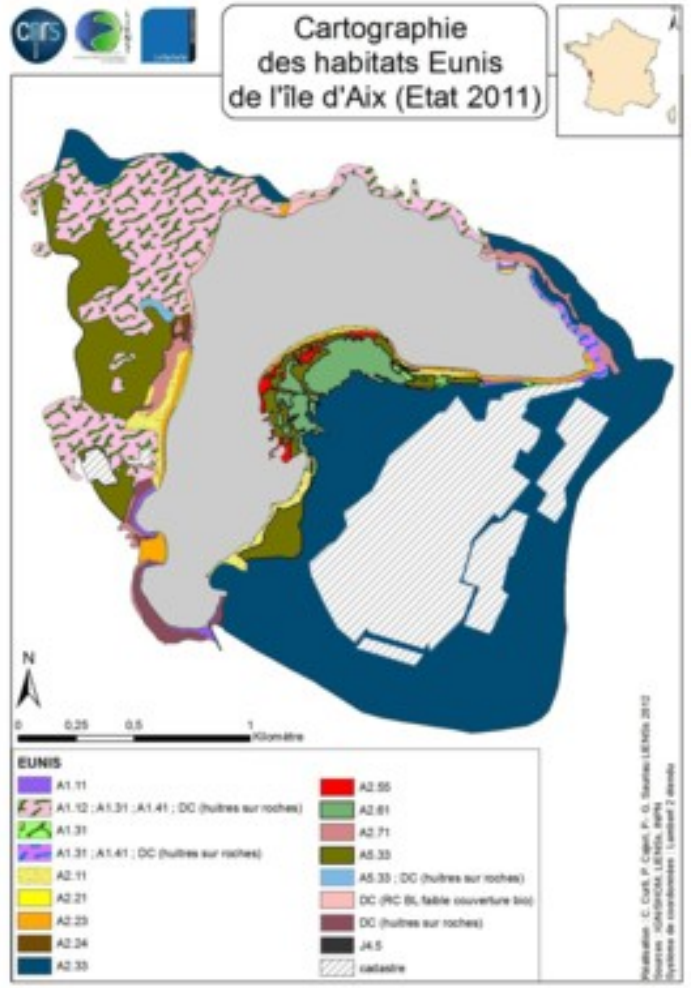

B

Figure 3. Schéma de traitement combinant vérités terrain (points limite et station), ortho littorales, scènes satellite pixélisées après analyses par arbre hiérarchique (A) ; cartographie EUNIS des habitats intertidaux de l'île d'Aix (B).

Sont aussi présents des habitats remarquables avec des espèces d'intérêt patrimonial comme l'herbier de Zostera noltii (A2.61) dans l'Anse du Saillant, non répertorié jusqu'alors (TARDY \& TARDY, 1977 ; AUBY et al., 2010), recouvrant une surface de $0,097 \mathrm{~km}^{2}$ ainsi que des récifs de Sabellaria alveolata (A2.71) sur la Grande Plage mais également le long du pourtour rocheux de l'île pour une surface totale de 0,056 km². 


\section{XII ${ }^{\text {èmes }}$ Journées Nationales Génie Côtier - Génie Civil \\ Cherbourg, 12-14 juin 2012}

La partie rocheuse de l'île est couverte de mosaïques comportant des ceintures algales à Fucales de milieu semi-abrité et à rhodophycées de milieu semi-exposé entremêlées de mares permanentes à forte diversité biologique. Ces mosaïques d'habitats sont utilisées en cartographie lorsque des habitats élémentaires sont imbriqués les uns dans les autres sans réelle possibilité de les individualiser à l'échelle du rendu cartographique. La mosaïque 2, par exemple, regroupe des ceintures algales à rhodophycées (A1.12) et à Fucales (A1.31), des mares permanentes (A1.41) ainsi qu'un habitat devenu très répandu, l'habitat intertidal "huîtres sur roches" à Crassostrea gigas. Elle met en avant l'imbrication de milieux abrité (A1.31) et exposé à semi-exposé (A1.12) sur le large pourtour Nord et Ouest de l'île vraisemblablement en relation avec les variations locales de l'hydrodynamisme généré par la houle et les mers de vent. Les peuplements de macroalgues sur Aix se caractérisent en effet par des variations très localisées de compositions spécifiques (LAHONDÈRE, 1984) combinant des milieux abrités et battus, généralement décrits comme distincts sur d’autres côtes.

Tableau 1. Surface relative des habitats intertidaux de l'île d'Aix.

\begin{tabular}{lll}
\hline Habitat & Code EUNIS & Surface $\left.\mathbf{( k m}^{2}\right)$ \\
\hline Bancs de moules et/ou de cirripèdes & $A 1.11$ & 0,019 \\
Ceintures de Fucales et/ou d'algues rouges & $A 1.12$ & intégré mosaïque 2 \\
Fucales sur côtes abritées & $A 1.31$ & 0,009 \\
Mares permanentes & $A 1.41$ & intégré mosaïques 2 et 3 \\
Galets et cailloutis intertidaux & $A 2.11$ & 0,056 \\
Sable fin de haut de plage & $A 2.21$ & 0,016 \\
Sables fins intertidaux à dominance Polychètes/amphipodes & $A 2.23$ & 0,042 \\
Sables vaseux intertidaux à dominance Polychètes/bivalves & $A 2.24$ & 0,005 \\
Vase marine intertidale & $A 2.33$ & 2,132 \\
Espèces pionnières des prés salés (Salicornes, Spartines) & $A 2.55$ & 0,017 \\
Herbiers à Zostera noltii & $A 2.61$ & 0,097 \\
Récifs à Sabellaria alveolata & $A 2.71$ & 0,056 \\
Vase sableuse infralittorale & $A 5.33$ & 0,323 \\
Ouvrages portuaires autres qu'immeubles & J4.5 & 0,001 \\
Cadastre conchylicole (pression anthropique ?) & - & 0,021 \\
Huîtres sur roches & création de code & 0,040 \\
Roches et blocs à faible couverture macrobiotique & création de code & 0,018 \\
A5.33 + huîtres sur roches & mosaïque 1 & 0,006 \\
A1.12 + A1.31 + A1.41 + huîtres sur roches & mosaïque 2 & 0,525 \\
A1.31+ A1.41 + huîtres sur roches & mosaïque 3 & 0,017 \\
\hline
\end{tabular}

Les pressions anthropiques exercées sur les habitats et espèces sont liées notamment à l'ostréiculture, la mytiliculture, la plaisance, au tourisme estival et à la pêche à pied. Cependant, l'île d'Aix profite de particularismes insulaires, comme l'absence de véhicules motorisés ou un éco-tourisme très développé. Bénéficiant de cette gestion, les espèces d'intérêt patrimonial sont considérées en bon état de conservation. Les récifs en boule d'hermelles de la Grande Plage forment des structures très développées et 
l'herbier de Zostera noltii présente des pourcentages de recouvrement élevés (mars n’étant que le début de la reprise de végétation) sans évidence de dégradation.

\subsection{Applicabilité de la typologie EUNIS}

L'exhaustivité de la typologie EUNIS a pu être mise en défaut lors de l'interprétation des vérités terrain (tableau 1). En regard des spécificités régionales, certains habitats n’y sont pas décrits de façon explicite tout comme ils sont absents des cahiers d'habitats (BENSETTITI et al., 2004). Pour pallier ce manque, des demandes de création de nouveaux habitats EUNIS seront formulées auprès du coordinateur MNHN-Paris, certaines étant déjà suggérées par GUILLAUMONT et al. (2009) et BAJJOUK et al. (2010) pour les côtes de Bretagne. De même, la logique de considérer les emprises du cadastre conchylicole (huître et moule) comme une pression anthropique se superposant aux habitats sous-jacents peut être inversée en considérant que ces activités génèrent 1) de nouveaux complexes d'habitats favorables à l'installation une faune et flore originale d'épibiontes sessiles sur roches et/ou substrats artificiels et 2) des perturbations modérées des substrats meubles sous-jacents et de leurs biocénoses (BOUCHET \& SAURIAU, 2008). Cette conceptualisation concorde avec la description de complexes d'habitats du littoral mélangeant substrats meubles et substrats rocheux. Il pourrait aussi être suggéré une analogie avec l'agriculture ou l'horticulture terrestre non-intensive dont les habitats relèvent du domaine I de la typologie EUNIS (DAVIES et al., 2004). De fait, la culture de l'huître sur estran s'apparente à un jardinage extensif sans apport d'intrant ni de phytosanitaire dans un milieu contraint avant tout par les aléas naturels, le regard de l'ethnologue sur la production de cette activité la qualifiant même de "moisson des marins-paysans" (LEGUÉ DUPONT, 2004).

\subsection{Bilan des avancées méthodologiques}

Les ortho littorales 2000 sont le support de base à la réalisation des polygones d'habitats mais les signatures colorimétriques des différents habitats à cartographier ne peuvent pas toujours être distinguées (GOURMELON \& ROBIN, 2005). L'association de cette source d'information avec les analyses des scènes KOMPSAT améliore la pertinence de la cartographie. En effet, la couverture spatiale associée à une résolution élevée des images ainsi qu'un étalonnage en grandeurs physiques de la mesure confèrent à cet outil les qualités nécessaires à la caractérisation biogéophysique des surfaces observées en intertidal (LAFON et al., 2008) ; cette propriété a déjà été exploitée pour la cartographie des marais charentais (REGRAIN, 1980). Notre stratégie d'analyse, basée sur une classification hiérarchique, repose sur des critères simples de discrimination des faciès deux à deux (présence d'eau révélée dans le canal proche infrarouge, indice de végétation normalisé pour localiser la végétation). Elle permet de maîtriser les habitats à déterminer et de traiter un ensemble d'images acquises à diverses dates. Les algorithmes et seuils de décision ne sont néanmoins pas optimisés mais permettent une cartographie 
correcte d'au moins $90 \%$ des cas. La mise en évidence des spartines reste une difficulté liée à une résolution trop faible de capteurs. Les difficultés de discrimination entre vases sableuses et sables vaseux sont reconnues car très proches radiométriquement (DEHOUCK et al., comm. pers. 2011). Les vérités terrain, les analyses bio-sédimentires et l'analyse préalable de la continuité des structures visibles sur orthophotographies permettent de lever ces biais.

\section{Conclusions}

Les acquis antérieurs à la cartographie des estrans d'Aix comportaient des inventaires d'espèces (DE MONTAUDOUIN \& SAURIAU, 2000), des descriptions ponctuelles du patrimoine naturel marin (TARDY \& TARDY, 1977; LAHONDÈRE, 1984) ou des cartes biosédimentaires des fonds meubles (HILY, 1976). Notre étude permet de combler un vide majeur de connaissances en particulier pour les habitats rocheux et les habitats d'espèces. La carte d'habitats proposée n'est cependant pas définitive. Elle devrait bénéficier d'ajouts apportés à la typologie EUNIS par l'incorporation de nouveaux habitats en cours de validation à l'échelle européenne.

\section{Références bibliographiques}

AUBY I., OGER-JEANNERET H., SAURIAU P.-G., HILY C., BARILLE L. (2010). Angiospermes des côtes françaises Manche-Atlantique. Propositions pour un indicateur DCE et premières estimations de la qualité. Ifremer, $72 \mathrm{p}+$ annexes.

BAJJOUK T., DERRIEN S., GENTIL F., HILY C., GRALL J. (2010). Typologie d'habitats marins benthiques : analyse de l'existant et propositions pour la cartographie. Habitats côtiers de la région Bretagne - Note de synthèse $n^{\circ} 2$, Habitats $d u$ circalittoral. Projets REBENT-Bretagne et Natura 2000-Bretagne. RST/IFREMER/DYNECO/AG/11-03/TB, 24 p + Annexes.

BENSETTITI F., BIORET F., ROLAND J., LACOSTE J.-P., GÉHU J.-M., GLÉMAREC M., BELLAN-SANTINI D. (eds.) (2004). Cahiers d'habitats Natura 2000. Connaissance et gestion des habitats et des espèces d'intérêt communautaire. Tome 2 Habitats côtiers. MATE/MAP/MNHN, $\mathrm{n}^{\circ}$ 2. La Documentation française, Paris, $399 \mathrm{p}$.

BOUCHET V.M.P., SAURIAU P.-G. (2008). Influence of oyster culture practices and environmental conditions on the ecological status of intertidal mudflats in the Pertuis Charentais (SW France): a multi-index approach. Marine Pollution Bulletin, $\mathrm{n}^{\circ}$ 56, pp 1898-1912.

DAVIES C.E., MOSS D., HILL M.O. (2004). EUNIS habitat classification. Revised 2004. Report to the European Topic Centre on Nature Protection and Biodiversity, European Environment Agency, 307 p.

DAUVIN J.-C. (ed.) (1997). Les biocénoses marines et littorales françaises des côtes atlantiques, Manche et Mer du Nord, Patrimoines Naturels / Série Patrimoine 
Ecologique, $n^{\circ}$ 28. Service du Patrimoine Naturel / IEGB) / Muséum National d'Histoire Naturelle, Paris, 376 p.

DE MONTAUDOUIN X., SAURIAU P.-G. (2000). Contribution to a synopsis of marine species richness in the Pertuis Charentais Sea with new insights in soft-bottom macrofauna of the Marennes-Oléron Bay. Cahiers de Biologie Marine, $n^{\circ} 41$, pp 181222.

GOURMELON F., ROBIN M. (2005). SIG et littoral. Lavoisier, Paris, 328 p.

GUILLAUMONT B., BAJJOUK T., ROLLET C., HILY C., GENTIL F. (2009). Typologie d'habitats marins benthiques : analyse de l'existant et propositions pour la cartographie (Habitats côtiers de la région Bretagne)- Note de synthèse, Projets REBENT-Bretagne et Natura-Bretagne. RST/IFREMER/DYNECO/AG/0806/REBENT, 22 p. + Tableaux.

HARDEGEN M., GOURMELON F., BIORET F., MAGNANON S. (2001). La cartographie des habitats terrestres du réseau Natura 2000 en Bretagne. Mappemonde, $n^{\circ}$ 64, pp 19-23.

HILY C. (1976). Ecologie benthique des pertuis Charentais. Thèse de $3^{\text {ème }}$ Cycle, Université de Bretagne Occidentale, 236 p.

LAFON V., MARIEU V., BUTEL R., DEHOUCK A., FROIDEFOND, J.-M., TRUT G. (2008). Cartographie des faciès bio-sédimentaires du Bassin d'Arcachon à partir de l'imagerie FORMOSAT-2. Actes Xèmes Journées Nationales Génie Côtier Génie Civil, 14-16 octobre 2008, Sofia Antipolis, pp 563-272. doi:10.5150/jngcgc.2008.054-L

LAHONDÈRE C. (1984). Compte rendu de la sortie algologique du 12 juin 1983 à l'île d'Aix. Bulletin de la Société Botanique du Centre-Ouest, n 15, pp 327-328.

LEGUÉ DUPONT P. (2004). La moisson des marins-paysans. Editions de la Maison des sciences de l'homme \& INRA, Paris, 308 p.

MARTY P., VIVIEN F.-D., LEPART J., LARRÈRE R. (2005). Les biodiversités objets, théories, pratiques. CNRS Editions, $261 \mathrm{p}$.

PINTON F., ALPHANDERY P., BILLAUD J.-P., DEVERRE C., FORTIER A., GÉNIAUX G. (2007). La construction du réseau Natura 2000 en France. La Documentation française, Paris, $249 \mathrm{p}$.

PROJET MESH (2008). Guide de cartographie des habitats marins. RST DYNECO/AG/07-20/JP, Ifremer, Centre de Brest, 342 p.

REGRAIN R. (1980). Géographie physique et télédétection des marais charentais. Paillart, Abbeville, 512 p.

ROLLET C., BONNOT-COURTOIS C., FOURNIER J. (2005). Cartographie des habitats benthiques en zone intertidale à partir des orthophotographies littorales. Fiche technique 13 - Projet REBENT. 16 p.

TARDY J., TARDY B. (1977). Le peuplement de la zone des marées du littoral. In : L'île d'Aix. Annales de la Société des Sciences Naturelles de la Charente-Maritime, Supplément, pp 167-172. 